\title{
Regularity of minimizers of autonomous convex variational integrals
}

\author{
Menita Carozza, Jan Kristensen and Antonia Passarelli di Napoli
}

\begin{abstract}
We establish local higher integrability and differentiability results for minimizers of variational integrals

$$
\mathfrak{F}(v, \Omega)=\int_{\Omega} F(D v(x)) \mathrm{d} x
$$

over $\mathrm{W}^{1, p}$-Sobolev mappings $v: \Omega \subset \mathbb{R}^{n} \rightarrow \mathbb{R}^{N}$ satisfying a Dirichlet boundary condition. The integrands $F$ are assumed to be autonomous, convex and of $(p, q)$ growth, but are otherwise not subjected to any further structure conditions, and we consider exponents in the range $1<p \leq q<p^{*}$, where $p^{*}$ denotes the Sobolev conjugate exponent of $p$.
\end{abstract}

Mathematics Subject Classification (2010): 49N15 (primary); 49N60, 49N99 (secondary).

\section{Introduction and statement of the results}

We prove local higher integrability and differentiability results for minimizers of the basic autonomous and convex variational integrals

$$
\mathfrak{F}(v, O)=\int_{O} F(D v(x)) \mathrm{d} x
$$

with integrands $F$ satisfying $(p, q)$ growth conditions, defined for mappings $v: \Omega \rightarrow \mathbb{R}^{N}$ of Sobolev class $\mathrm{W}^{1, p}$ and open subsets $O$ of a fixed bounded and open subset $\Omega$ of $\mathbb{R}^{n}$. Our results mainly concern the multi-dimensional vectorial case $n, N \geq 2$, but some aspects are also new in the multi-dimensional scalar case, $n \geq 2$ and $N=1$. The one-dimensional case, $n=1$ and $N \geq 1$, is special and stronger results apply there.

Parts of the research were done while MC and APdN were visiting the Oxford Centre for Nonlinear PDE, and while JK was visiting Dept. Maths. 'R. Caccioppoli' in Napoli. We wish to thank both institutions for financial support and hospitality. The work was supported by the EPSRC Science and Innovation award to the Oxford Centre for Nonlinear PDE (EP/E035027/1), and JK was also partially supported by ERC grant 207573 'Vectorial Problems'.

Received July 24, 2012; accepted in revised form September 25, 2012. 
In order to state and describe the results we consider an integrand $F: \mathbb{R}^{N \times n} \rightarrow$ $\mathbb{R}$ satisfying the following growth and convexity hypotheses:

$$
\begin{gathered}
F(\xi) \leq L\left(|\xi|^{q}+1\right) \\
\xi \mapsto F(\xi)-\ell\left(\mu^{2}+|\xi|^{2}\right)^{\frac{p}{2}} \quad \text { is convex }
\end{gathered}
$$

for all $\xi \in \mathbb{R}^{N \times n}$. Here $L, \ell>0$ and $\mu \geq 0$ are arbitrary but fixed constants, whereas the exponents $q \geq p>1$ will be subjected to various constraints.

Hypothesis (H2) is a uniform strong $p$-convexity condition for the integrand $F$, and is similar to the condition considered in [10]. In fact, when $F$ is $\mathrm{C}^{2}$ then $(\mathrm{H} 2)$ is equivalent to the following standard strong $p$-ellipticity condition

$$
F^{\prime \prime}(\xi)[\eta, \eta] \geq c\left(\mu^{2}+|\xi|^{2}\right)^{\frac{p-2}{2}}|\eta|^{2}
$$

for all $\xi, \eta \in \mathbb{R}^{N \times n}$, where $c$ is a positive constant of form $c=c(p) \ell$. While when $F$ is $\mathrm{C}^{1}$ the hypothesis (H2) is equivalent to the following standard strong $p$-monotonicity condition

$$
\left\langle F^{\prime}(\xi)-F^{\prime}(\eta), \xi-\eta\right\rangle \geq c\left(\mu^{2}+|\xi|^{2}+|\eta|^{2}\right)^{\frac{p-2}{2}}|\xi-\eta|^{2}
$$

for all $\xi, \eta \in \mathbb{R}^{N \times n}$, where again $c=\tilde{c}(p) \ell$. It is easy to see that hypothesis (H2) in particular implies that the integrand $F$ is bounded from below, and we shall therefore often implicitly assume that $F$ is nonnegative once (H2) holds.

It is well-known that for convex integrands, the growth condition (H1) implies a Lipschitz condition, which for $\mathrm{C}^{1}$ integrands can be stated as

$$
\left|F^{\prime}(\xi)\right| \leq c\left(|\xi|^{q-1}+1\right)
$$

for all $\xi \in \mathbb{R}^{N \times n}$, where we can use $c=2^{q} L$.

We will be concerned with two closely related classes of $F$-minimizers of the variational integral (1.1), under the assumptions $(\mathrm{H} 1),(\mathrm{H} 2)$, which are defined as follows.

Definition 1.1. (i) A mapping $u \in \mathrm{W}^{1, p}\left(\Omega, \mathbb{R}^{N}\right)$ is an $F$-minimizer if $F(D u) \in$ $\mathrm{L}^{1}(\Omega)$ and

$$
\int_{\Omega} F(D u) \leq \int_{\Omega} F(D v)
$$

for all $v \in \mathrm{W}_{u}^{1, p}\left(\Omega, \mathbb{R}^{N}\right)$.

(ii) A mapping $u \in \mathrm{W}_{\text {loc }}^{1,1}\left(\Omega, \mathbb{R}^{N}\right)$ is a local $F$-minimizer if $F(D u) \in \mathrm{L}_{\text {loc }}^{1}(\Omega)$ and

$$
\int_{O} F(D u) \leq \int_{O} F(D v)
$$

for any $O \Subset \Omega$ and any $v \in \mathrm{W}_{u}^{1, p}\left(O, \mathbb{R}^{N}\right)$. 
Here we use the notation $O \Subset \Omega$ as a short-hand for: $O$ is an open set whose closure, $\bar{O}$, is compact and contained in $\Omega$. Furthermore for an open subset $O \subset \Omega$ (no regularity of $\partial O$ implied) and $u \in \mathrm{W}^{1, p}\left(O, \mathbb{R}^{N}\right)$ we denote by $\mathrm{W}_{u}^{1, p}\left(O, \mathbb{R}^{N}\right)$ the Dirichlet class of $\mathrm{W}^{1, p}$ Sobolev maps $v$ such that the difference $v-u \in$ $\mathrm{W}_{0}^{1, p}\left(O, \mathbb{R}^{N}\right)$, where the latter is defined to be the closure of the space of smooth and compactly supported test maps, $\mathrm{C}_{c}^{\infty}\left(O, \mathbb{R}^{N}\right)$, in $\mathrm{W}^{1, p}\left(O, \mathbb{R}^{N}\right)$. We refer to the monograph [29] for background theory on the relevant function spaces.

We emphasize that in the definitions of $F$-minimality it is crucial for regularity theory that we include the integrability requirements on $F(D u)$.

The assumptions $(\mathrm{H} 1),(\mathrm{H} 2)$ clearly entail a $(p, q)$ growth condition: there exists a constant $c=c(L / \ell, p, q, \mu)>0$ such that

$$
\frac{1}{c}|\xi|^{p}-c \leq F(\xi) \leq c\left(|\xi|^{q}+1\right)
$$

for all $\xi \in \mathbb{R}^{N \times n}$.

A systematic study of the regularity of minimizers of such functionals was initiated with the celebrated papers by Marcellini (see in particular [19-21]). From the beginning it has been clear that no regularity can be expected if the coercitivity and growth exponents, denoted $p$ and $q$, respectively, are too far apart (see $[13,15$, $18]$ and also $[9,11])$. On the other hand, many regularity results are available if the ratio $q / p$ is bounded above by a suitable constant that in general depends on the dimension $n$, and converges to 1 when $n$ tends to infinity (incl. [1-3,7-9,22,24]).

In the present context of $p$-convex integrands of $q$-growth the higher differentiability of minimizers is obtained by a variant of the difference-quotient method in connection with some sort of regularization procedure (see in particular [7-9] and [3]). In particular we emphasize that all such results rely heavily on the strong convexity hypothesis imposed on the integrand, and that this allows for a treatment based on (a suitable version of) the Euler-Lagrange system. However it should be noted that for a direct derivation of the Euler-Lagrange system we already have to require that the exponents $p$ and $q$ are sufficiently close. Indeed, it is well-known that for a convex $\mathrm{C}^{1}$ integrand $F$ satisfying (1.3) with exponents $q \leq p+1$ the $F$ minimality of a $\mathrm{W}^{1, p}$-map $u$ implies that it is an $F$-extremal too: the field $F^{\prime}(D u)$ is locally integrable (in fact, locally $p /(q-1)$-integrable by (1.2)) and is row-wise solenoidal in the sense that

$$
\int_{\Omega}\left\langle F^{\prime}(D u), D \varphi\right\rangle=0
$$

for all $\varphi \in \mathrm{C}_{c}^{\infty}\left(\Omega, \mathbb{R}^{N}\right)$. Using a regularization procedure and convex duality theory we shall establish much stronger results in Proposition 3.1 below.

The main results of this paper are the local higher integrability result stated in Theorem 1.2 and the local higher differentiability result stated in Theorem 1.3. Both results concern minimizers of the integral functional (1.1) under the assumptions (H1) and (H2). We obtain local higher integrability for minimizers when the 
exponents $p$ and $q$ satisfy the condition $1<p \leq q<p^{*}$, where $p^{*}$ denotes the Sobolev conjugate exponent of $p$. The interpretation of this is

$$
\begin{gathered}
1<p \leq q<\frac{n p}{n-p} \text { when } p<n \\
1<p \leq q<\infty \text { when } p \geq n .
\end{gathered}
$$

We emphasize that as opposed to many of the above mentioned results, we do not require any additional structure assumption on the integrand. More precisely we have the following:

Theorem 1.2. Let $F: \mathbb{R}^{N \times n} \rightarrow \mathbb{R}$ be $\mathrm{C}^{1}$ and satisfy (H1), (H2) with $\mu=0$. Assume

$$
1<p \leq q<p^{*}
$$

where $p^{*}$ denotes the Sobolev conjugate exponent of $p$ (with the interpretation of (1.4)). For $g \in \mathrm{W}^{1, q}\left(\Omega, \mathbb{R}^{N}\right)$ let $u \in \mathrm{W}_{g}^{1, p}\left(\Omega, \mathbb{R}^{N}\right)$ be the unique $F$-minimizer. Then $u \in \mathrm{W}_{\text {loc }}^{1, q}\left(\Omega, \mathbb{R}^{N}\right)$ when $q<\frac{n p}{n-1}$, and $u \in \mathrm{W}_{\text {loc }}^{1, r}\left(\Omega, \mathbb{R}^{N}\right)$ for all $r<\bar{p}$, where

$$
\bar{p}:=\frac{n p}{n-\frac{p}{p-1}\left(1-n\left(\frac{1}{p}-\frac{1}{q}\right)\right)} \quad \text { when } \quad q \geq \frac{n p}{n-1} .
$$

The proof of Theorem 1.2 is based on the difference-quotient method but, in contrast to the above mentioned papers, under our assumptions on the exponents $p$ and $q$, we can not use directly that an $F$-minimizer is a solution to the corresponding Euler-Lagrange system. Instead we approximate the integrand $F$ by suitably regular integrands in order to facilitate a systematic use of the dual problems in the sense of Convex Analysis. Namely we approximate $F$ by strictly convex and uniformly elliptic integrands $F_{k}$, satisfying standard $p$-growth conditions, whose minimizers $u_{k}$ strongly converge to the minimizer $u$ in $\mathrm{W}^{1, p}$. To every such minimizer $u_{k}$ we can associate, essentially according to the standard duality theory for convex problems [6], a row-wise solenoidal matrix field denoted by $\sigma_{k}$. For the pair $\left(D u_{k}, \sigma_{k}\right)$ we shall establish suitable estimates, that are preserved in passing to the limit. Such estimates will provide conditions in order for the Euler-Lagrange system to hold for an $F$-minimizer, as well as a first regularity result (see Proposition 3.1 in Section 3 ). While the dual problems have been used previously in regularity theory, see for instance $[4,5]$, it seems that the observations used here have so far not been employed in the anisotropic growth context. We refer the reader to Remark 3.3 for a discussion of the somewhat mysterious exponent $\bar{p}$ that appears in Theorem 1.2. As a consequence of the higher integrability result of Theorem 1.2, we are to able to establish the following

Theorem 1.3. Assume $F: \mathbb{R}^{N \times n} \rightarrow \mathbb{R}$ is $\mathrm{C}^{1}$ and satisfies (H1), (H2) for some exponents $1<p \leq q<\infty$. Let $u \in \mathrm{W}_{\mathrm{loc}}^{1, p}\left(\Omega, \mathbb{R}^{N}\right)$ be a local $F$-minimizer. Setting

$$
V(D u):=\left(\mu^{2}+|D u|^{2}\right)^{\frac{p-2}{4}} D u,
$$


we have that

$$
V(D u) \in \mathrm{W}_{\text {loc }}^{1,2}\left(\Omega, \mathbb{R}^{N \times n}\right),
$$

provided $q<\frac{n p}{n-1}$.

We remark that under the assumptions $(\mathrm{H} 1),(\mathrm{H} 2)$ on the integrand $F$ the $\mathrm{W}_{\text {loc }}^{1,2}$ higher differentiability of $V(D u)$ has previously been established only when the exponents $p, q$ satisfy the stronger conditions $1<p \leq q<\frac{n+1}{n} p$, see [8], and also $[2,3,7,9,10]$. Our improved bound $q<\frac{n p}{n-1}$ is in a certain sense more natural as it also appears in connection with optimal trace and embedding results for Sobolevtype spaces. Namely when $B \subset \mathbb{R}^{n}$ is an open ball (or any smooth bounded domain), then any $h \in \mathrm{W}^{1, p}(\partial B)$ can be extended to $H \in \mathrm{W}^{1, q}(B)$ precisely for $q \leq \frac{n p}{n-1}$. See for instance [28] for such trace and embedding theorems.

Finally, we remark that our results can be generalized to minimizers of the general autonomous convex variational integral

$$
\int_{\Omega} F(v, D v) \mathrm{d} x
$$

The precise statements and proofs are left to the interested reader, and we only note that it is essential that the integrand $F=F(y, z)$ be jointly convex for the validity of the results. Similar remarks, together with precise statements and sketches of proofs, were given in [3].

\section{Preliminaries}

In this paper we follow the usual convention and denote by $c$ a general constant that may vary on different occasions, even within the same line of estimates. Relevant dependencies on parameters and special constants will be suitably emphasized using parentheses or subscripts. All the norms we use on $\mathbb{R}^{n}, \mathbb{R}^{N}$ and $\mathbb{R}^{N \times n}$ will be the standard euclidean ones and denoted by $|\cdot|$ in all cases. In particular, for matrices $\xi, \eta \in \mathbb{R}^{N \times n}$ we write $\langle\xi, \eta\rangle:=\operatorname{trace}\left(\xi^{T} \eta\right)$ for the usual inner product of $\xi$ and $\eta$, and $|\xi|:=\langle\xi, \xi\rangle^{\frac{1}{2}}$ for the corresponding euclidean norm. When $a \in \mathbb{R}^{N}$ and $b \in \mathbb{R}^{n}$ we write $a \otimes b \in \mathbb{R}^{N \times n}$ for the tensor product defined as the matrix that has the element $a_{r} b_{s}$ in its r-th row and s-th column. Observe that $(a \otimes b) x=(b \cdot x) a$ for $x \in \mathbb{R}^{n}$, and $|a \otimes b|=|a||b|$.

When $F: \mathbb{R}^{N \times n} \rightarrow \mathbb{R}$ is sufficiently differentiable, we write

$$
F^{\prime}(\xi)[\eta]:=\left.\frac{\mathrm{d}}{\mathrm{d} t}\right|_{t=0} F(\xi+t \eta) \quad \text { and } \quad F^{\prime \prime}(\xi)[\eta, \eta]:=\left.\frac{\mathrm{d}^{2}}{\mathrm{~d} t^{2}}\right|_{t=0} F(\xi+t \eta)
$$

for $\xi, \eta \in \mathbb{R}^{N \times n}$. Hereby we think of $F^{\prime}(\xi)$ both as an $N \times n$ matrix and as the corresponding linear form on $\mathbb{R}^{N \times n}$, though $\left|F^{\prime}(\xi)\right|$ will always denote the euclidean norm of the matrix $F^{\prime}(\xi)$. The second derivative, $F^{\prime \prime}(\xi)$, is a real bilinear 
form on $\mathbb{R}^{N \times n}$. We express growth conditions for the second derivative of the integrand in terms of the operator norm on bilinear forms:

$$
\left\|F^{\prime \prime}(\xi)\right\|:=\sup _{|\eta| \leq 1,|\zeta| \leq 1} F^{\prime \prime}(\xi)[\eta, \zeta] .
$$

It is convenient, and by now common, to express the convexity and growth conditions for the integrands in terms of two auxiliary functions defined for all $\xi \in \mathbb{R}^{N \times n}$ as

$$
\langle\xi\rangle=\langle\xi\rangle_{\mu}:=\left(\mu^{2}+|\xi|^{2}\right)^{\frac{1}{2}}
$$

and

$$
V(\xi)=V_{p, \mu}(\xi):=\left(\mu^{2}+|\xi|^{2}\right)^{\frac{p-2}{4}} \xi,
$$

where $\mu \geq 0$ and $p \geq 1$ are parameters. For the auxiliary function $V_{p, \mu}$, we record the following estimate (see the proof of [14, Lemma 8.3]):

Lemma 2.1. Let $1<p<\infty$ and $0 \leq \mu \leq 1$. There exists a constant $c=$ $c(n, N, p)>0$ such that

$$
c^{-1}\left(\mu^{2}+|\xi|^{2}+|\eta|^{2}\right)^{\frac{p-2}{2}} \leq \frac{\left|V_{p, \mu}(\xi)-V_{p, \mu}(\eta)\right|^{2}}{|\xi-\eta|^{2}} \leq c\left(\mu^{2}+|\xi|^{2}+|\eta|^{2}\right)^{\frac{p-2}{2}}
$$

for any $\xi, \eta \in \mathbb{R}^{N \times n}$.

We shall use a class of fractional Sobolev spaces that can be defined in terms of Nikolskii conditions. For a vector valued function $w: A \rightarrow \mathbb{R}^{k}$, a natural number $1 \leq s \leq n$ and a real number $h \in \mathbb{R}$, we define the finite difference operator

$$
\Delta_{s, h} w(x):=w\left(x+h e_{s}\right)-w(x),
$$

where $\left\{e_{1}, \ldots, e_{n}\right\}$ denotes the canonical basis of $\mathbb{R}^{n}$. Note that hereby $\Delta_{s, h} w(x)$ is well-defined whenever $x, x+h e_{s} \in A$.

Definition 2.2. Let $A \subset \mathbb{R}^{n}$ be an open set, $k \in \mathbb{N}, \alpha \in(0,1)$ and $q \in[1, \infty)$. For a mapping $w \in \mathrm{L}_{\text {loc }}^{q}\left(A, \mathbb{R}^{k}\right)$ we say that $w$ is locally in $\mathrm{B}_{\infty}^{\alpha, q}$ on $A$ provided for each ball $B \Subset A$ there exist $d \in(0, \operatorname{dist}(B, \partial A)), M>0$ such that

$$
\int_{B}\left|\Delta_{s, h} w(x)\right|^{q} \mathrm{~d} x \leq M|h|^{q \alpha}
$$

for every $s \in\{1, \ldots, n\}$ and $h \in \mathbb{R}$ satisfying $|h| \leq d$.

Theorem 2.3. On any domain $\Omega \subset \mathbb{R}^{n}$ we have the continuous embeddings:

(i) $\mathrm{B}_{\infty}^{\alpha, q} \hookrightarrow \mathrm{L}_{\mathrm{loc}}^{r}$ for all $r<\frac{n q}{n-\alpha q}$ provided $\alpha \in(0,1), q>1$ and $\alpha q<n$;

(ii) $\mathrm{W}_{\text {loc }}^{1, p} \hookrightarrow \mathrm{B}_{\infty}^{\alpha, q}$ provided $\alpha=1-n\left(\frac{1}{p}-\frac{1}{q}\right)$, where $1<p \leq q<\infty$. 
We refer to [28, Sections 30-32] for a proof of this theorem. In fact, the above statements follow by localizing the corresponding results proved for functions defined on $\mathbb{R}^{n}$ in [28] by simply using a smooth cut-off function.

We shall require some further elementary notions from convex analysis, all of which are discussed in the scalar case $N=1$ in [6]. However, as we shall briefly demonstrate below, the relevant parts easily extend to the vectorial case $N>1$ too. Let $F: \mathbb{R}^{N \times n} \rightarrow \mathbb{R}$ satisfy the $(p, q)$ growth condition:

$$
c_{1}|\xi|^{p}-c_{2} \leq F(\xi) \leq c_{2}\left(|\xi|^{q}+1\right),
$$

where $0<c_{1} \leq c_{2}$ and $1<p \leq q<\infty$. Its polar (or Fenchel conjugate) integrand is defined by

$$
F^{*}(\zeta):=\sup _{\xi \in \mathbb{R}^{N \times n}}(\langle\zeta, \xi\rangle-F(\xi)), \quad \zeta \in \mathbb{R}^{N \times n}
$$

Hereby $F^{*}: \mathbb{R}^{N \times n} \rightarrow \mathbb{R}$ is convex and has $\left(q^{\prime}, p^{\prime}\right)$ growth, where $p^{\prime}, q^{\prime}$ are the Hölder conjugate exponents of $p, q$, respectively. More precisely, as can readily be checked, we have

$$
c_{3}|\zeta|^{q^{\prime}}-c_{2} \leq F^{*}(\zeta) \leq c_{4}|\zeta|^{p^{\prime}}+c_{2}
$$

for all $\zeta \in \mathbb{R}^{N \times n}$, where $c_{3}=c_{2}^{-\frac{1}{q-1}}\left(1-\frac{1}{q}\right) q^{-\frac{1}{q-1}}$ and $c_{4}=c_{1}^{-\frac{1}{p-1}}\left(1-\frac{1}{p}\right) p^{-\frac{1}{p-1}}$. One can check that the bipolar integrand $F^{* *}:=\left(F^{*}\right)^{*}$ equals $F$ at $\xi$ if and only if $F$ is lower semicontinuous and convex at $\xi$, and more generally, that it is the convex envelope of $F$. In particular, $F^{* *}=F$ precisely when $F$ is convex and lower semicontinuous (the latter being a consequence of the former when, as here, $F$ is real-valued).

The definition of polar integrand means that we have the Young-type inequality

$$
\langle\zeta, \xi\rangle \leq F^{*}(\zeta)+F^{* *}(\xi)
$$

for all $\zeta, \xi \in \mathbb{R}^{N \times n}$. Notice that for a given $\xi$ we have equality in (2.5) precisely for $\zeta \in \partial F^{* *}(\xi)$, the subgradient for $F^{* *}$ at $\xi$. Furthermore, we record that $F$ is strictly convex precisely when $F^{*}$ is $\mathrm{C}^{1}$, and that in this case we also have

$$
\left(F^{*}\right)^{\prime}\left(F^{\prime}(\xi)\right)=\xi
$$

for all $\xi \in \mathbb{R}^{N \times n}$.

We now specialize to integrands satisfying standard $p$-growth and convexity conditions, and so assume that $F: \mathbb{R}^{N \times n} \rightarrow \mathbb{R}$ is a $C^{1}$ function satisfying

$$
|F(\xi)| \leq L\left(|\xi|^{p}+1\right)
$$

and

$$
\xi \mapsto F(\xi)-\ell|\xi|^{p} \quad \text { is convex }
$$


where $0<\ell \leq L<\infty$ and $1<p<\infty$. Note that (2.7) is (H1) with $\mu=1$ ( $q=p$ and slightly larger $L$ ) and (2.8) is (H2) with $\mu=0$. The polar integrand $F^{*}: \mathbb{R}^{N \times n} \rightarrow \mathbb{R}$ is then strictly convex and $\mathrm{C}^{1}$, and it satisfies the $p^{\prime}$-growth condition:

$$
c_{1}|\zeta|^{p^{\prime}}-c_{2} \leq F^{*}(\zeta) \leq c_{2}\left(|\zeta|^{p^{\prime}}+1\right)
$$

for all $\zeta \in \mathbb{R}^{N \times n}$, where $c_{1}=c_{1}(L, p)>0, c_{2}=c_{2}(\ell, p)>0$ and $p^{\prime}=$ $p /(p-1)$. By standard arguments, for a given $g \in \mathrm{W}^{1, p}\left(\Omega, \mathbb{R}^{N}\right)$, the problem of minimizing $\int_{\Omega} F(D v)$ over $v \in \mathrm{W}_{g}^{1, p}\left(\Omega, \mathbb{R}^{N}\right)$ admits a unique solution $u$. This minimizer is also the unique weak solution $u \in \mathrm{W}_{g}^{1, p}\left(\Omega, \mathbb{R}^{N}\right)$ to the EulerLagrange system:

$$
\int_{\Omega}\left\langle F^{\prime}(D u), D \varphi\right\rangle=0
$$

for all $\varphi \in \mathrm{W}_{0}^{1, p}\left(\Omega, \mathbb{R}^{N}\right)$. In view of the Young-type inequality (2.5), and the subsequent remark, we have for the minimizer $u$ the extremality relation:

$$
\left\langle F^{\prime}(D u), D u\right\rangle=F^{*}\left(F^{\prime}(D u)\right)+F(D u)
$$

valid pointwise almost everywhere on $\Omega$. Hence for any row-wise solenoidal field $\sigma \in \mathrm{L}^{p^{\prime}}\left(\Omega, \mathbb{R}^{N \times n}\right)$ it follows that

$$
\int_{\Omega}\left(\left\langle F^{\prime}(D u), D u\right\rangle-F^{*}\left(F^{\prime}(D u)\right)\right) \geq \int_{\Omega}\left(\langle\sigma, D u\rangle-F^{*}(\sigma)\right) .
$$

Because $u-g \in \mathrm{W}_{0}^{1, p}\left(\Omega, \mathbb{R}^{N}\right)$ and $\sigma$ is row-wise solenoidal and $p^{\prime}$-integrable, we have $\int_{\Omega}\langle\sigma, D u\rangle=\int_{\Omega}\langle\sigma, D g\rangle$. Consequently, $F^{\prime}(D u)$ is the unique maximizer of the functional

$$
\sigma \mapsto \int_{\Omega}\left(\langle\sigma, D g\rangle-F^{*}(\sigma)\right)
$$

over all row-wise solenoidal fields $\sigma \in \mathrm{L}^{p^{\prime}}\left(\Omega, \mathbb{R}^{N \times n}\right)$. The extremality relation (2.9) can also be expressed in terms of $\sigma^{*}:=F^{\prime}(D u)$ and then reads as

$$
\left\langle\sigma^{*},\left(F^{*}\right)^{\prime}\left(\sigma^{*}\right)\right\rangle=F^{*}\left(\sigma^{*}\right)+F\left(\left(F^{*}\right)^{\prime}\left(\sigma^{*}\right)\right),
$$

where $\left(F^{*}\right)^{\prime}\left(\sigma^{*}\right)=D u$ being row-wise curl-free is merely a restatement of the Euler-Lagrange equation for the maximization problem of the functional (2.10) over solenoidal fields.

\section{Proof of Theorem 1.2}

Throughout this section we let $F: \mathbb{R}^{N \times n} \rightarrow \mathbb{R}$ be a $C^{1}$ integrand satisfying

$$
\xi \mapsto F(\xi)-\ell|\xi|^{p} \quad \text { is convex }
$$


and

$$
0 \leq F(\xi) \leq c\left(|\xi|^{q}+1\right)
$$

where $1<p \leq q<\infty$ and $\ell, c>0$ are constants. We shall impose additional conditions on the exponents $p$ and $q$ as we go along. It is a routine matter to check that $\mathfrak{F}(v, \Omega)=\int_{\Omega} F(D v)$ under the assumptions (3.1), (3.2) is a strictly convex, lower semicontinuous and proper functional on $\mathrm{W}^{1, p}\left(\Omega, \mathbb{R}^{N}\right)$. Hence for a given $g \in \mathrm{W}^{1, p}\left(\Omega, \mathbb{R}^{N}\right)$ with $\mathfrak{F}(g, \Omega)<\infty$ the existence and uniqueness of a minimizer $u$ in the Dirichlet class $\mathrm{W}_{g}^{1, p}\left(\Omega, \mathbb{R}^{N}\right)$ is then evident.

We split the proof of Theorem 1.2 in two parts and start with the following preliminary result. We state it as a separate result because we believe it could have independent interest. It gives conditions for the Euler-Lagrange system to hold for the minimizer $u$, and also contains a first higher integrability result.

Proposition 3.1. Assume $F: \mathbb{R}^{N \times n} \rightarrow \mathbb{R}$ is $\mathrm{C}^{1}$ and satisfies (3.1), (3.2). For $g \in$ $\mathrm{W}^{1, p}\left(\Omega, \mathbb{R}^{N}\right)$ with $F(D g) \in \mathrm{L}^{1}(\Omega)$, let $u \in \mathrm{W}_{g}^{1, p}\left(\Omega, \mathbb{R}^{N}\right)$ denote the unique $F$ minimizer. We then have the following two statements (where $F^{*}$ denotes the polar of $F$ ):

(i) If $g \in \mathrm{W}^{1, q}\left(\Omega, \mathbb{R}^{N}\right)$, then $F^{*}\left(F^{\prime}(D u)\right) \in \mathrm{L}^{1}(\Omega)$ and $F^{\prime}(D u)$ is row-wise solenoidal.

(ii) If $q \leq \frac{n p}{n-1}$, then $F^{*}\left(F^{\prime}(D u)\right) \in \mathrm{L}_{\mathrm{loc}}^{1}(\Omega)$ and $F^{\prime}(D u)$ is row-wise solenoidal.

Hence in both cases (i)-(ii), $u$ is in particular an $F$-extremal and $F^{\prime}(D u) \in$ $\mathrm{L}^{q^{\prime}}\left(\Omega, \mathbb{R}^{N \times n}\right)$, where $q^{\prime}=q /(q-1)$.

Remark 3.2. Note that in (i) above, apart from $1<p \leq q<\infty$, we do not impose any conditions on the exponents $p$ and $q$. Furthermore, as the reader can observe from the proof below, we in fact establish that $\sigma^{*}:=F^{\prime}(D u)$ is the unique maximizer of the dual problem that consists in maximizing the functional

$$
\sigma \mapsto \int_{\Omega}\left(\langle D g, \sigma\rangle-F^{*}(\sigma)\right)
$$

over row-wise solenoidal fields $\sigma \in \mathrm{L}^{q^{\prime}}\left(\Omega, \mathbb{R}^{N \times n}\right)$, where $F^{*}$ denotes the polar integrand of $F$ and $q^{\prime}=q /(q-1)$.

Proof. We start by constructing a class of auxiliary problems, whose solutions on the one hand approximate the minimizer $u$, and on the other can be dealt with by standard means. The construction might seem a bit elaborate at first, but all properties that we establish play a role in the proof, though some only at a later stage of the proof of the main result. Put

$$
G(\xi):=F(\xi)-\frac{\ell}{2}|\xi|^{p}
$$


$\xi \in \mathbb{R}^{N \times n}$. Then $G-\frac{\ell}{2}|\cdot|^{p}$ is convex by (3.1) so that in particular $G(\xi)-\frac{\ell}{2}|\xi|^{p} \geq$ $G(0)+\left\langle G^{\prime}(0), \xi\right\rangle$ for all $\xi$, and hence invoking also (3.2) we find a (new) constant $c>0$ such that

$$
c\left(|\xi|^{q}+1\right) \geq G(\xi) \geq \frac{1}{c}|\xi|^{p}-c
$$

for all $\xi \in \mathbb{R}^{N \times n}$. Consequently, the polar of $G$,

$$
G^{*}(z):=\sup _{\xi \in \mathbb{R}^{N \times n}}(\langle\xi, z\rangle-G(\xi))
$$

is a real-valued convex function satisfying a $\left(q^{\prime}, p^{\prime}\right)$ growth condition, where $q^{\prime}, p^{\prime}$ denote the Hölder conjugate exponents of $q, p$, respectively (compare with (2.4)).

For each $k>0$ define

$$
G_{k}(\xi):=\max _{|z| \leq k}\left(\langle\xi, z\rangle-G^{*}(z)\right) .
$$

Then $G_{k}$ is a real-valued convex, globally $k$-Lipschitz function, and because $G$ is (lower semicontinuous and) convex we have that

$$
G_{k}(\xi) \nearrow G^{* *}(\xi)=G(\xi) \quad \text { as } \quad k \nearrow \infty
$$

pointwise in $\xi \in \mathbb{R}^{N \times n}$. Define

$$
\tilde{G}_{k}(\xi):=\max \left\{G_{k}(\xi), \frac{1}{c}|\xi|^{p}-c\right\}
$$

In view of (3.3) we still have that $\tilde{G}_{k}(\xi) \nearrow G(\xi)$ as $k \nearrow \infty$. Since $p>1$ and $G_{k}$ is $k$-Lipschitz there exist numbers $r_{k}>0$ such that $r_{k} \nearrow \infty$ as $k \nearrow \infty$ and $\tilde{G}_{k}(\xi)=\frac{1}{c}|\xi|^{p}-c$ for $|\xi| \geq r_{k}-1$. Define

$$
H_{k}(\xi):= \begin{cases}\tilde{G}_{k}(\xi) & \text { when }|\xi| \leq r_{k} \\ \frac{p}{c} r_{k}^{p-1}|\xi|-\frac{p-1}{c} r_{k}^{p}-c & \text { when }|\xi|>r_{k} .\end{cases}
$$

It is not hard to check that $H_{k}$ is convex and globally $m_{k}$-Lipschitz (we may take any $m_{k} \geq \frac{p}{c} r_{k}^{p-1}$ ). Moreover,

$$
H_{k}(\xi) \nearrow G^{* *}(\xi)=G(\xi) \quad \text { as } \quad k \nearrow \infty
$$

pointwise in $\xi \in \mathbb{R}^{N \times n}$. Next we regularize $H_{k}$ by use of the following standard radially symmetric and smooth convolution kernel

$$
\Phi(\xi):= \begin{cases}c \exp \left(\frac{1}{|\xi|^{2}-1}\right) & \text { for }|\xi|<1 \\ 0 & \text { for }|\xi| \geq 1\end{cases}
$$


where the constant $c=c(n N)$ is chosen such that $\int_{\mathbb{R}^{N \times n}} \Phi=1$, and for each $\varepsilon>0$ we put $\Phi_{\varepsilon}(\xi):=\varepsilon^{-n N} \Phi\left(\varepsilon^{-1} \xi\right)$. It is routine to check that the mollified function $\Phi_{\varepsilon} * H_{k}$ (as usual defined by convolution) is convex and $\mathrm{C}^{\infty}$, and since $H_{k}$ is convex and $m_{k}$-Lipschitz that

$$
H_{k}(\xi) \leq\left(\Phi_{\varepsilon} * H_{k}\right)(\xi) \leq H_{k}(\xi)+m_{k} \varepsilon
$$

holds for all $\xi \in \mathbb{R}^{N \times n}$. For integers $k>1$ and sequences $\left(\delta_{k}\right),\left(\mu_{k}\right) \subset(0, \infty)$ (specified at (3.7) below), we define

$$
F_{k}(\xi):=\left(\Phi_{\delta_{k}} * H_{k}\right)(\xi)-\mu_{k}+\frac{\ell}{2}|\xi|^{p}
$$

Then we have for all $\xi \in \mathbb{R}^{N \times n}$ and $k>1$ :

$$
\begin{aligned}
F_{k}(\xi) & \leq H_{k}(\xi)+m_{k} \delta_{k}-\mu_{k}+\frac{\ell}{2}|\xi|^{p} \\
& \leq H_{k+1}(\xi)+m_{k} \delta_{k}-\mu_{k}+\frac{\ell}{2}|\xi|^{p} \\
& \leq\left(\Phi_{\delta_{k+1}} * H_{k+1}\right)(\xi)+m_{k} \delta_{k}-\mu_{k}+\frac{\ell}{2}|\xi|^{p} \\
& =F_{k+1}(\xi)+\mu_{k+1}+m_{k} \delta_{k}-\mu_{k} .
\end{aligned}
$$

where we used (3.5) and the monotonicity of the sequence $H_{k}$. Hence taking

$$
\delta_{k}:=\frac{1}{k^{2} m_{k}} \text { and } \mu_{k}:=\frac{1}{k-1}
$$

we have that $F_{k}(\xi) \nearrow F(\xi)$ as $k \nearrow \infty$ pointwise in $\xi$. It follows in particular from Dini's Lemma that the convergence is locally uniform in $\xi$. We note that $F_{k}$ is $\mathrm{C}^{1}$ on $\mathbb{R}^{N \times n}, \mathrm{C}^{\infty}$ on $\mathbb{R}^{N \times n} \backslash\{0\}$, and that it is $\mathrm{C}^{2}$ on $\mathbb{R}^{N \times n}$ when $p \geq 2$. Next we check that also $F_{k}^{\prime}(\xi) \rightarrow F^{\prime}(\xi)$ locally uniformly in $\xi$ as $k \rightarrow \infty$. To that end assume that $\xi_{k} \rightarrow \xi$ and consider $\left(F_{k}^{\prime}\left(\xi_{k}\right)\right)$. Because difference-quotients of convex functions are increasing in the increment, we have for all $\eta \in \mathbb{R}^{N \times n}$ and $0<|t| \leq 1$ :

$$
\begin{aligned}
\left|\left\langle F_{k}^{\prime}\left(\xi_{k}\right)-F^{\prime}(\xi), \eta\right\rangle\right| & \leq\left|\frac{F_{k}\left(\xi_{k}+t \eta\right)-F_{k}\left(\xi_{k}\right)-\left\langle F^{\prime}(\xi), t \eta\right\rangle}{t}\right| \\
& \leq\left|F_{k}\left(\xi_{k}+\eta\right)-F_{k}\left(\xi_{k}\right)-\left\langle F^{\prime}(\xi), \eta\right\rangle\right|
\end{aligned}
$$

Consequently, we have for all $\eta \in \mathbb{R}^{N \times n}$ that

$$
\limsup _{k \rightarrow \infty}\left|\left\langle F_{k}^{\prime}\left(\xi_{k}\right)-F^{\prime}(\xi), \eta\right\rangle\right| \leq\left|F(\xi+\eta)-F(\xi)-\left\langle F^{\prime}(\xi), \eta\right\rangle\right|
$$


and since $F$ in particular is differentiable at $\xi$ we conclude that the left-hand side must vanish. This proves the asserted local uniform convergence of derivatives. Finally, we also record that $F_{k}-\frac{\ell}{2}|\cdot|^{p}$ is convex, that

$$
\left\|F_{k}^{\prime \prime}(\xi)\right\| \leq c_{k}\left(|\xi|^{p-2}+1\right)
$$

for all $\xi \in \mathbb{R}^{N \times n} \backslash\{0\}$, where $c_{k}$ are positive real constants (possibly $c_{k} \nearrow \infty$ of course). It is also easy to see that $F_{k}$ satisfy a uniform $(p, q)$ growth condition.

Let $u_{k} \in \mathrm{W}_{g}^{1, p}\left(\Omega, \mathbb{R}^{N}\right)$ denote the unique $F_{k}$-minimizer, and recall from above that the row-wise solenoidal matrix field $\sigma_{k}:=F_{k}^{\prime}\left(D u_{k}\right) \in \mathrm{L}^{p^{\prime}}\left(\Omega, \mathbb{R}^{N \times n}\right)$ is a solution to the dual problem that consists in maximizing the functional

$$
\int_{\Omega}\left(\langle\sigma, D g\rangle-F_{k}^{*}(\sigma)\right)
$$

over row-wise solenoidal matrix fields $\sigma \in \mathrm{L}^{p}\left(\Omega, \mathbb{R}^{N \times n}\right)$, where $F_{k}^{*}$ denotes the polar of $F_{k}$. As the $F_{k}$ satisfy a uniform $(p, q)$ growth condition, the $F_{k}^{*}$ satisfy a uniform $\left(q^{\prime}, p^{\prime}\right)$ growth condition, and it is not difficult to check that $F_{k}^{*}(\zeta) \searrow$ $F^{*}(\zeta)$ as $k \nearrow \infty$ pointwise in $\zeta$. Furthermore, we record the extremality relation

$$
\left\langle\sigma_{k}, D u_{k}\right\rangle=F_{k}^{*}\left(\sigma_{k}\right)+F_{k}\left(D u_{k}\right) \quad \text { a.e. on } \Omega
$$

that holds for all $k>1$, and that, since $\sigma_{k} \in \mathrm{L}^{p^{\prime}}$ is row-wise solenoidal and $u_{k}-g \in$ $\mathrm{W}_{0}^{1, p}\left(\Omega, \mathbb{R}^{N}\right)$,

$$
\int_{\Omega}\left\langle\sigma_{k}, D u_{k}\right\rangle=\int_{\Omega}\left\langle\sigma_{k}, D g\right\rangle
$$

Our next goal is to show that $u_{k} \rightarrow u$ strongly in $\mathrm{W}^{1, p}$. To that end, we start by observing that

$$
\int_{\Omega}\left(\frac{1}{c}\left|D u_{k}\right|^{p}-c\right) \leq \int_{\Omega} F_{k}\left(D u_{k}\right) \leq \int_{\Omega} F(D g)<\infty
$$

so $\left(u_{k}\right)$ is bounded in $\mathrm{W}^{1, p}\left(\Omega, \mathbb{R}^{N}\right)$. Let $\left(u_{k^{\prime}}\right)$ be a subsequence. Then by the reflexivity of $\mathrm{W}^{1, p}$, it admits a further subsequence $\left(u_{k^{\prime \prime}}\right)$ that converges weakly to some $v$ in $\mathrm{W}^{1, p}$. By Mazur's lemma, $\mathrm{W}_{g}^{1, p}\left(\Omega, \mathbb{R}^{N}\right)$ is also $\mathrm{W}^{1, p}$-weakly closed, so $v \in \mathrm{W}_{g}^{1, p}\left(\Omega, \mathbb{R}^{N}\right)$, and relabelling the subsequence we write simply $u_{k} \rightarrow v$. Now, by Mazur's Lemma, we get for each $k>1$

$$
\liminf _{j \rightarrow \infty} \int_{\Omega} F_{k}\left(D u_{j}\right) \geq \int_{\Omega} F_{k}(D v),
$$

and since $F_{k} \nearrow F$, we find by monotone convergence and minimality of $u$

$$
\liminf _{k \rightarrow \infty} \int_{\Omega} F_{k}\left(D u_{k}\right) \geq \int_{\Omega} F(D v) \geq \int_{\Omega} F(D u) .
$$


Using first that $u_{k}$ is $F_{k}$-minimizing, and then monotone convergence, yield

$$
\limsup _{k \rightarrow \infty} \int_{\Omega} F_{k}\left(D u_{k}\right) \leq \limsup _{k \rightarrow \infty} \int_{\Omega} F_{k}(D u)=\int_{\Omega} F(D u),
$$

and by comparing this with the foregoing inequalities we deduce that

$$
\int_{\Omega} F_{k}\left(D u_{k}\right) \rightarrow \int_{\Omega} F(D u)=\int_{\Omega} F(D v) .
$$

By uniqueness of $F$-minimizers, $v=u$. To deduce that the convergence is actually strong we use the uniform $p$-convexity of the $F_{k}$, we have that $F_{k}-\frac{\ell}{2}|\cdot|{ }^{p}$ is convex for all $k>1$. So, as $F_{k}$ is $\mathrm{C}^{1}$, we deduce from Lemma 2.1 that there exists a constant $c>0$ such that

$$
c|V(\xi)-V(\eta)|^{2} \leq F_{k}(\xi)-F_{k}(\eta)-\left\langle F_{k}^{\prime}(\eta), \xi-\eta\right\rangle
$$

holds for all $\xi, \eta \in \mathbb{R}^{N \times n}$ and $k>1$. Here $V(\xi)=V_{p, 0}(\xi)=|\xi|^{\frac{p-2}{2}} \xi$. Consequently,

$$
\begin{aligned}
c \int_{\Omega}\left|V(D u)-V\left(D u_{k}\right)\right|^{2} & \leq \int_{\Omega}\left(F_{k}(D u)-F_{k}\left(D u_{k}\right)-\left\langle F_{k}^{\prime}\left(D u_{k}\right), D\left(u-u_{k}\right)\right\rangle\right) \\
& =\int_{\Omega}\left(F_{k}(D u)-F_{k}\left(D u_{k}\right)\right) \rightarrow 0
\end{aligned}
$$

as $k \rightarrow \infty$. It follows that $D u_{k} \rightarrow D u$ in measure on $\Omega$ and that $\left|V\left(D u_{k}\right)\right|^{2}=\left|D u_{k}\right|^{p}$ is equi-integrable on $\Omega$, hence, by Vitali's convergence theorem, that $D u_{k} \rightarrow D u$ strongly in $\mathrm{L}^{p}$. Since $u_{k}-u \in \mathrm{W}_{0}^{1, p}\left(\Omega, \mathbb{R}^{N}\right)$ we have shown that the (relabelled) subsequence $\left(u_{k}\right)$ converges strongly to $u$ in $\mathrm{W}^{1, p}$. By the uniqueness of limit we conclude by a standard argument that the full sequence $\left(u_{k}\right)$ converges strongly in $\mathrm{W}^{1, p}$ to $u$. It follows in particular that $\sigma_{k}=F_{k}^{\prime}\left(D u_{k}\right) \rightarrow F^{\prime}(D u)$ in measure on $\Omega$, and so passing to the limit in (3.9) we recover, with $\sigma^{*}:=F^{\prime}(D u)$, the pointwise extremality relation

$$
\left\langle\sigma^{*}, D u\right\rangle=F^{*}\left(\sigma^{*}\right)+F(D u) \quad \text { a.e. on } \Omega .
$$

Up to this point we have not used any of the conditions on the boundary datum $g$ or on the exponents $p, q$ listed in (i)-(ii). We now assume that $g \in \mathrm{W}^{1, q}\left(\Omega, \mathbb{R}^{N}\right)$ corresponding to (i). Then in view of (3.9), (3.10) and the uniform $\left(q^{\prime}, p^{\prime}\right)$ growth of $F_{k}^{*}$ we deduce that $\left(\sigma_{k}\right)$ is bounded in $\mathrm{L}^{q^{\prime}}\left(\Omega, \mathbb{R}^{N \times n}\right)$. Namely,

$$
\begin{aligned}
\int_{\Omega}\left(\frac{1}{c}\left|\sigma_{k}\right|^{q^{\prime}}-c\right) & \leq \int_{\Omega} F_{k}^{*}\left(\sigma_{k}\right)=\int_{\Omega}\left\langle\sigma_{k}, D u_{k}\right\rangle-F_{k}\left(D u_{k}\right) \\
& =\int_{\Omega}\left\langle\sigma_{k}, D g\right\rangle-F_{k}\left(D u_{k}\right) \\
& \leq \frac{c}{2} \int_{\Omega}\left|\sigma_{k}\right|^{q^{\prime}}+c \int_{\Omega}|D g|^{q}+\int_{\Omega} F_{k}\left(D u_{k}\right)
\end{aligned}
$$


and hence

$$
\int_{\Omega}\left|\sigma_{k}\right|^{q^{\prime}} \leq c\left(\int_{\Omega}|D g|^{q}+\int_{\Omega} F_{k}\left(D u_{k}\right)\right)
$$

Therefore, by Fatou's lemma and by (3.11), we have that

$$
\left\|F^{\prime}(D u)\right\|_{\mathrm{L}^{q^{\prime}}}^{q^{\prime}} \leq \liminf _{k \rightarrow \infty}\left\|\sigma_{k}\right\|_{\mathrm{L}^{q^{\prime}}}^{q^{\prime}} \leq c\left(\int_{\Omega}|D g|^{q}+\int_{\Omega} F(D u)\right) .
$$

As it is then clear that $F^{\prime}(D u)$ is row-wise solenoidal this proves (i). Finally, regarding Remark 3.2, note that in view of (3.9), (3.10) the field $\sigma^{*}$ is a maximizer of

$$
\sigma \mapsto \int_{\Omega}\left(\langle D g, \sigma\rangle-F^{*}(\sigma)\right)
$$

over row-wise solenoidal fields $\sigma \in \mathrm{L}^{q^{\prime}}\left(\Omega, \mathbb{R}^{N \times n}\right)$. By strict convexity it is then the unique such maximizer.

We next turn to (ii), and assume that $q \leq n p /(n-1)$. Since $u \in \mathrm{W}^{1, p}\left(\Omega, \mathbb{R}^{N}\right)$ we can for each $x_{0} \in \Omega$ find a ball $B=B\left(x_{0}, R\right) \subset \Omega$ such that $\left.u\right|_{\partial B} \in$ $\mathrm{W}^{1, p}\left(\partial B, \mathbb{R}^{N}\right)$, see for instance [29]. If $h$ denotes the harmonic extension of $\left.u\right|_{\partial B}$ to $B$, then it is well-known that $h \in \mathrm{W}^{1, \frac{n p}{n-1}}\left(B, \mathbb{R}^{N}\right)$. We can now repeat the above argument for (i), where this time we define the auxiliary minimizers $u_{k}$ with $B, h$ substituted for $\Omega, g$, respectively.

Now, we are going to prove Theorem 1.2 from the Introduction. The key new point in the proof is that we estimate the field $\sigma^{*}=F^{\prime}(D u)$ using Proposition 3.1 rather than merely by use of the (consequence of the) growth condition (1.2). The outcome is a better higher integrability estimate for the minimizer. The remaining parts of the proof are standard in the present context, and consist of a differencequotient argument applied in the setting of fractional Sobolev spaces (compare for instance [9]) and the regularized problems defined in the proof of Proposition 3.1.

Proof of Theorem 1.2: Conclusion. Define the integrands $F_{k}$ and corresponding $F_{k}$-minimizers $u_{k}$ of class $\mathrm{W}_{g}^{1, p}\left(\Omega, \mathbb{R}^{N}\right)$ as in the proof of Proposition 3.1 (see in particular (3.6)). We have shown there that $u_{k} \rightarrow u$ strongly in $\mathrm{W}^{1, p}$ and that $\sigma_{k}:=F_{k}^{\prime}\left(D u_{k}\right) \rightarrow F^{\prime}(D u)$ weakly in $\mathrm{L}^{q^{\prime}}$ and in measure on $\Omega$. Furthermore, $F_{k}-\frac{\ell}{2}|\cdot|^{p}$ is convex and

$$
\int_{\Omega}\left\langle F_{k}^{\prime}\left(D u_{k}\right), D \varphi\right\rangle=0
$$

for all $\varphi \in \mathrm{W}_{0}^{1, p}\left(\Omega, \mathbb{R}^{N}\right)$. We shall establish uniform integrability bounds on $\left(u_{k}\right)$ to conclude the proof. Recall that the auxiliary $V$-function for the degenerate case $\mu=0$ is defined as $V\left(D u_{k}\right)=V_{p, 0}\left(D u_{k}\right)=\left|D u_{k}\right|^{\frac{p-2}{2}} D u_{k}$. 
Fix $B_{3 R}=B\left(x_{0}, 3 R\right) \subset \Omega$, an integer $1 \leq s \leq n$, and an increment $0 \neq h \in$ $(-R, R)$. It follows that

$$
\int_{B_{2 R}}\left\langle\Delta_{s, h} F_{k}^{\prime}\left(D u_{k}\right), D \varphi\right\rangle=0
$$

for all $\varphi \in \mathrm{W}_{0}^{1, p}\left(B_{2 R}, \mathbb{R}^{N}\right)$. In particular we may take $\varphi=\theta \Delta_{s, h} u_{k}$ for $\theta \in$ $\mathrm{C}_{c}^{1}\left(B_{2 R}\right)$, whereby

$$
\int_{B_{2 R}}\left\langle\Delta_{s, h} F_{k}^{\prime}\left(D u_{k}\right), \Delta_{s, h} D u_{k}\right\rangle \theta=-\int_{B_{2 R}}\left\langle\Delta_{s, h} F_{k}^{\prime}\left(D u_{k}\right), \Delta_{s, h} u_{k} \otimes D \theta\right\rangle
$$

follows. Consequently, taking $\theta$ nonnegative and so $\theta=1$ on $B_{R}$ we may use $\left(\mathrm{H} 2^{\prime \prime}\right.$ ) (since $F_{k}$ is $\mathrm{C}^{1}$ ), Lemma 3 and Hölder's inequality to find a constant $c>0$, which in particular is independent of $k$, such that

$$
\begin{aligned}
\int_{B_{R}}\left|\Delta_{s, h} V\left(D u_{k}\right)\right|^{2} & \leq c\left(\int_{B_{3 R}}\left|\sigma_{k}\right|^{q^{\prime}}\right)^{\frac{1}{q^{\prime}}}\left(\int_{B_{2 R}}\left|\Delta_{s, h} u_{k}\right|^{q}\right)^{\frac{1}{q}} \sup |D \theta| \\
& \leq \tilde{c}\left(\int_{B_{2 R}}\left|\Delta_{s, h} u_{k}\right|^{q}\right)^{\frac{1}{q}},
\end{aligned}
$$

where

$$
\tilde{c}:=c \sup _{k}\left(\int_{B_{3 R}}\left|\sigma_{k}\right|^{q^{\prime}}\right)^{\frac{1}{q^{\prime}}} \sup |D \theta|
$$

is finite according to Proposition 3.1. Now to extract information from this estimate, we recall that $\left(u_{k}\right)$ in particular is bounded in $\mathrm{W}^{1, p}$, and that by the version (ii) of the Sobolev Embedding stated in Theorem 2.3, $\mathrm{W}_{\text {loc }}^{1, p} \hookrightarrow \mathrm{B}_{\infty}^{\alpha, q}$ boundedly, provided $\alpha=1-n\left(\frac{1}{p}-\frac{1}{q}\right)$. The condition (1.5) on $q$ guarantees that $\alpha \in(0,1]$. Divide (3.16) by $|h|^{\alpha}$, and infer from the arbitrariness of the ball $B$, the direction $s$ and the increment $h$, that $\left(V\left(D u_{k}\right)\right)$ is bounded in $\mathrm{B}_{\infty}^{\frac{\alpha}{2}, 2}$ locally on $\Omega$. Now by version (i) of the Sobolev Embedding stated in Theorem 2.3, we have that $\mathrm{B}_{\infty}^{\frac{\alpha}{2}, 2} \hookrightarrow \mathrm{L}_{\text {loc }}^{r}$ boundedly for each $r<\frac{2 n}{n-\alpha}$. Therefore $\left(D u_{k}\right)$ is bounded in $\mathrm{L}_{\text {loc }}^{r}$ for each $r<\frac{n p}{n-\alpha}$ and hence $\left(u_{k}\right)$ is bounded in $\mathrm{W}_{\text {loc }}^{1, r}$ for each $r<\frac{n p}{n-\alpha}$.

We can now repeat the above estimation using this improved bound on $\left(u_{k}\right)$. The details are as follows. Put

$$
p_{0}:=p, \quad p_{j}:=\frac{n p}{n-1+n\left(\frac{1}{p_{j-1}}-\frac{1}{q}\right)}
$$

for $j \in \mathbb{N}$. Observe that we can rewrite the exponent $\bar{p}$ at (1.6) as

$$
\bar{p}=\frac{n p}{n-\frac{p}{p-1}\left(1-n\left(\frac{1}{p}-\frac{1}{q}\right)\right)}=\frac{n(p-1)}{n-1-\frac{n}{q}},
$$


and that, for $p_{j-1}<\bar{p}$, we have $p_{j-1}<p_{j}<\bar{p}$. Because

$$
\bar{p}>p \quad \text { precisely when } q<p^{*},
$$

a straightforward calculation yields that

$$
p_{j} \nearrow \bar{p} \quad \text { as } \quad j \nearrow \infty \text {. }
$$

With these observations in place we apply the above difference-quotient argument to deduce that if $\left(u_{k}\right)$ is bounded in $\mathrm{W}_{\text {loc }}^{1, r}$ for each $r<p_{j-1}$ and $p \leq p_{j-1} \leq q$, then it is also bounded in $\mathrm{W}_{\text {loc }}^{1, r}$ for each $r<p_{j}$. In view of Remark 3.3 below it follows that, for $q<\frac{n p}{n-1}$, the sequence $\left(u_{k}\right)$ is bounded in $\mathrm{W}_{\text {loc }}^{1, q}$, while for $\frac{n p}{n-1} \leq q<p^{*}$ it is bounded in $\mathrm{W}_{\text {loc }}^{1, r}$ for all $r<\bar{p}$. The conclusion follows easily from this.

Remark 3.3. We record that

$$
p<\frac{n p}{n-\frac{p}{p-1}\left(1-n\left(\frac{1}{p}-\frac{1}{q}\right)\right)}<q \text { when } \quad \frac{n p}{n-1}<q<p^{*}
$$

and

$$
\frac{n p}{n-\frac{p}{p-1}\left(1-n\left(\frac{1}{p}-\frac{1}{q}\right)\right)} \geq q \quad \text { when } \quad p \leq q \leq \frac{n p}{n-1} \quad \text { and } \quad q>\frac{n}{n-1}
$$

Hence there is integrability improvement locally in $\Omega$ of $F$-minimizers for the full range of exponents $q$ satisfying (1.5). Furthermore, $\bar{p}=\frac{n p}{n-1}$ when $q=\frac{n p}{n-1}$, and $\bar{p}=\bar{p}(q)$ is decreasing as a function of $q$ with

$$
\begin{cases}\bar{p} \searrow p \text { as } q \nearrow p^{*} \quad \text { when } 1<p<n \\ \bar{p} \searrow \frac{n(p-1)}{n-1} \text { as } q \nearrow \infty & \text { when } p \geq n,\end{cases}
$$

where we remark that $\frac{n(p-1)}{n-1} \geq p$ for $p \geq n$ with equality precisely when $p=n$.

\section{Proof of Theorem 1.3}

Throughout this section $u \in \mathrm{W}_{\text {loc }}^{1, p}\left(\Omega, \mathbb{R}^{N}\right)$ denotes a local $F$-minimizer. For the sake of simplicity, we shall give the proof in case the integrand $F: \mathbb{R}^{N \times n} \rightarrow \mathbb{R}$ is $\mathrm{C}^{2}$ and satisfies the hypotheses (H1) and $\left(H 2^{\prime}\right)$, with $q<\frac{p n}{n-1}$. The general case can be treated by a suitable approximation argument, inspired by [10] and [8], and also sketched in [3]. 
Our aim is to show that $V(D u) \in \mathrm{W}_{\text {loc }}^{1,2}\left(\Omega, \mathbb{R}^{N \times n}\right)$, where we recall the definition of the auxiliary functions as

$$
V(\xi):=\langle\xi\rangle^{\frac{p-2}{2}} \xi, \quad\langle\xi\rangle:=\sqrt{\mu^{2}+|\xi|^{2}}
$$

For later reference we note that for a $\mathrm{C}^{2}$ map $w$ a routine calculation yields

$$
|D[V(D w)]|^{2} \leq\left(\frac{|p-2|}{2}+1\right)^{2}\langle D w\rangle^{p-2}\left|D^{2} w\right|^{2} .
$$

Before proceeding with the proof of Theorem 1.3, we need to carry out an approximation procedure, which is essentially based on the arguments contained in [3]. Here we give a version suitable for our needs, partly for the sake of completeness and partly because the present set-up differs slightly from that of [3].

Fix a subdomain with a smooth boundary $\Omega^{\prime} \Subset \Omega$ and take $k \in \mathbb{N}$, so large that we have the continuous embedding $\mathrm{W}^{k, 2}\left(\Omega^{\prime}\right) \hookrightarrow \mathrm{C}^{2}\left(\overline{\Omega^{\prime}}\right)$. For a smooth kernel $\phi \in \mathrm{C}_{c}^{\infty}\left(B_{1}(0)\right)$ with $\phi \geq 0$ and $\int_{B_{1}(0)} \phi=1$, we consider the corresponding family of mollifiers $\left(\phi_{\varepsilon}\right)_{\varepsilon>0}$ and put $\tilde{u}_{\varepsilon}:=\phi_{\varepsilon} * u$ on $\Omega^{\prime}$ for each positive $\varepsilon<\operatorname{dist}\left(\Omega^{\prime}, \partial \Omega\right)$. By Theorem 1.2, we have that $D u \in L_{\mathrm{loc}}^{q}$ and hence

$$
\tilde{u}_{\varepsilon} \rightarrow u \text { as } \varepsilon \searrow 0 \text { strongly in } \mathrm{W}^{1, q}\left(\Omega^{\prime}, \mathbb{R}^{N}\right) .
$$

Moreover we remark that, for a suitable function $\tilde{\varepsilon}=\tilde{\varepsilon}(\varepsilon)$ with $\tilde{\varepsilon} \searrow 0$ as $\varepsilon \searrow 0$, we also have

$$
\tilde{\varepsilon} \int_{\Omega^{\prime}}\left|D^{k} \tilde{u}_{\varepsilon}\right|^{2} \rightarrow 0 \text { as } \varepsilon \searrow 0 \text {. }
$$

For small $\varepsilon>0$, we let $u_{\varepsilon} \in \mathrm{W}^{k, 2}\left(\Omega^{\prime}\right) \cap \mathrm{W}_{\tilde{u}_{\varepsilon}}^{1, p}\left(\Omega^{\prime}\right)$ denote a minimizer to the functional

$$
v \mapsto \int_{\Omega^{\prime}}\left(F(D v)+\frac{\tilde{\varepsilon}}{2}\left|D^{k} v\right|^{2}\right)
$$

on the Sobolev class $\mathrm{W}^{k, 2}\left(\Omega^{\prime}\right) \cap \mathrm{W}_{\tilde{u}_{\varepsilon}}^{1, p}\left(\Omega^{\prime}\right)$. The existence of $u_{\varepsilon}$ is easily established by the direct method. Next two Lemmas are proven in [3] (see Lemmas 8 and 9 there) in a more general version. Here we state them in the form needed for our aims.

Lemma 4.1. For each $\varphi \in \mathrm{W}^{k, 2}\left(\Omega^{\prime}\right) \cap \mathrm{W}_{0}^{1, p}\left(\Omega^{\prime}\right)$,

$$
0=\int_{\Omega^{\prime}}\left(\left\langle F^{\prime}\left(D u_{\varepsilon}\right), D \varphi\right\rangle+\tilde{\varepsilon}\left\langle D^{k} u_{\varepsilon}, D^{k} \varphi\right\rangle\right) .
$$

Furthermore, $u_{\varepsilon} \in \mathrm{W}_{\mathrm{loc}}^{2 k, 2}\left(\Omega^{\prime}\right)$. 
Lemma 4.2. As $\varepsilon \searrow 0$, we have that

$$
\int_{\Omega^{\prime}}\left|D u_{\varepsilon}-D u\right|^{p} \mathrm{~d} x \rightarrow 0
$$

and

$$
\int_{\Omega^{\prime}} F\left(D u_{\varepsilon}\right) \mathrm{d} x \rightarrow \int_{\Omega^{\prime}} F(D u) \mathrm{d} x .
$$

We are now ready to prove Theorem 1.3.

Proof of Theorem 1.3. Fix $B_{2 R}=B_{2 R}\left(x_{0}\right) \subset \Omega^{\prime}$, radii $R \leq r<s \leq 2 R \leq 2$ and a smooth cut-off function $\rho$ satisfying $1_{B_{r}} \leq \rho \leq 1_{B_{s}}$ and $\left|D^{i} \rho\right| \leq\left(\frac{2}{s-r}\right)^{i}$ for each $i \in \mathbb{N}$. According to Lemma 4.1, we can test the Euler-Lagrange system (4.4) with $\varphi=\rho^{2 k} D_{j}^{2} u_{\varepsilon}$, for each direction $1 \leq j \leq n$, thus getting

$$
\begin{aligned}
0= & \int_{\Omega^{\prime}}\left\langle F^{\prime}\left(D u_{\varepsilon}\right), D_{j}^{2} D u_{\varepsilon}\right\rangle \rho^{2 k}+\int_{\Omega^{\prime}}\left\langle F^{\prime}\left(D u_{\varepsilon}\right), D_{j}^{2} u_{\varepsilon} \otimes D\left(\rho^{2 k}\right)\right\rangle \\
& +\tilde{\varepsilon} \int_{\Omega^{\prime}}\left\langle D^{k} u_{\varepsilon}, D^{k}\left(D_{j}^{2} u_{\varepsilon} \rho^{2 k}\right)\right\rangle \\
= & : I+I I+I I I .
\end{aligned}
$$

Integration by parts yields

$$
\begin{aligned}
I= & -\int_{\Omega^{\prime}}\left(\rho^{2 k} F^{\prime \prime}\left(D u_{\varepsilon}\right)\left[D_{j} D u_{\varepsilon}, D_{j} D u_{\varepsilon}\right]\right) \\
& -\int_{\Omega^{\prime}}\left(2 k\left\langle\rho^{2 k-1} D_{j} \rho F^{\prime}\left(D u_{\varepsilon}\right), D_{j} D u_{\varepsilon}\right\rangle\right) \\
\leq & -\int_{\Omega^{\prime}} \rho^{2 k}\left\langle D u_{\varepsilon}\right\rangle^{p-2}\left|D_{j} D u_{\varepsilon}\right|^{2}+2 k \int_{\Omega^{\prime}} \rho^{2 k-1}\left|D_{j} \rho\right|\left\langle D u_{\varepsilon}\right\rangle^{q-1}\left|D_{j} D u_{\varepsilon}\right|
\end{aligned}
$$

where we used assumptions (H2') and (1.2). Hence, using Young's inequality in the last integral, we obtain

$$
\begin{aligned}
I \leq & -\int_{\Omega^{\prime}} \rho^{2 k}\left\langle D u_{\varepsilon}\right\rangle^{p-2}\left|D_{j} D u_{\varepsilon}\right|^{2}+\frac{1}{2} \int_{\Omega^{\prime}} \rho^{2 k}\left\langle D u_{\varepsilon}\right\rangle^{p-2}\left|D_{j} D u_{\varepsilon}\right|^{2} \\
& +c(p, k) \int_{\Omega^{\prime}} \rho^{2(k-1)}\left|D_{j} \rho\right|^{2}\left\langle D u_{\varepsilon}\right\rangle^{2 q-p} \\
\leq & -\frac{1}{2} \int_{\Omega^{\prime}} \rho^{2 k}\left\langle D u_{\varepsilon}\right\rangle^{p-2}\left|D_{j} D u_{\varepsilon}\right|^{2}+c(p, k) \int_{\Omega^{\prime}} \rho^{2(k-1)}\left|D_{j} \rho\right|^{2}\left\langle D u_{\varepsilon}\right\rangle^{2 q-p} .
\end{aligned}
$$


Similarly, by virtue of (1.2) and Cauchy-Schwarz' inequality, we get

$$
\begin{aligned}
I I & \leq c(p, k) \int_{\Omega^{\prime}}\left\langle D u_{\varepsilon}\right\rangle^{q-1} \rho^{2 k-1}|D \rho|\left|D_{j}^{2} u_{\varepsilon}\right| \\
& \leq c(p, k) \int_{\Omega^{\prime}}\left\langle D u_{\varepsilon}\right\rangle^{2 q-p} \rho^{2(k-1)}|D \rho|^{2}+\frac{1}{4} \int_{\Omega^{\prime}} \rho^{2 k}\left\langle D u_{\varepsilon}\right\rangle^{p-2}\left|D u_{j} D u_{\varepsilon}\right|^{2} .
\end{aligned}
$$

In order to estimate $I I I$, we argue as in [3] writing

$$
I I I=\tilde{\varepsilon} \int_{\Omega^{\prime}}\left\langle D^{k} u_{\varepsilon}, D_{j} D^{k}\left(\rho^{2 k} D_{j} u_{\varepsilon}\right)-D^{k}\left(D_{j}\left(\rho^{2 k}\right) D_{j} u_{\varepsilon}\right)\right\rangle
$$

and integrating the first term by parts,

$$
\begin{aligned}
I I I & =-\tilde{\varepsilon} \int_{\Omega^{\prime}}\left(\left\langle D_{j} D^{k} u_{\varepsilon}, D^{k}\left(\rho^{2 k} D_{j} u_{\varepsilon}\right)\right\rangle-\tilde{\varepsilon} \int_{\Omega^{\prime}}\left\langle D^{k} u_{\varepsilon}, D^{k}\left(D_{j}\left(\rho^{2 k}\right) D_{j} u_{\varepsilon}\right)\right\rangle\right) \\
& =: I I I_{1}+I I I_{2} .
\end{aligned}
$$

We estimate these terms by use of Cauchy-Schwarz' inequality, Leibniz' product formula and the assumptions on $D^{i} \rho$ (simplifying also by use of $s-r \leq 1$ ):

$$
\begin{aligned}
I I I_{1} & \leq-\tilde{\varepsilon} \int_{\Omega^{\prime}} \rho^{2 k}\left|D_{j} D^{k} u_{\varepsilon}\right|^{2}+\frac{c_{k} \tilde{\varepsilon}}{(s-r)^{k}} \int_{\Omega^{\prime}} \rho^{k}\left|D_{j} D^{k} u_{\varepsilon}\right| \sum_{i=0}^{k-1}\left|D^{i} D_{j} u_{\varepsilon}\right| \\
& \leq-\frac{2 \tilde{\varepsilon}}{3} \int_{\Omega^{\prime}} \rho^{2 k}\left|D_{j} D^{k} u_{\varepsilon}\right|^{2}+\frac{c_{k} \tilde{\varepsilon}}{(s-r)^{2 k}} \int_{B_{2 R}}\left(\sum_{i=0}^{k-1}\left|D^{i} D_{j} u_{\varepsilon}\right|\right)^{2} \\
& \leq-\frac{2 \tilde{\varepsilon}}{3} \int_{\Omega^{\prime}} \rho^{2 k}\left|D_{j} D^{k} u_{\varepsilon}\right|^{2}+\frac{c_{k} \tilde{\varepsilon}}{(s-r)^{2 k}} \int_{B_{2 R}} \sum_{i=0}^{k-1}\left|D^{i} D_{j} u_{\varepsilon}\right|^{2}
\end{aligned}
$$

for a (new) constant $c_{k}$. Likewise,

$$
I I I_{2} \leq \frac{\tilde{\varepsilon}}{3} \int_{\Omega^{\prime}} \rho^{2 k}\left|D_{j} D^{k} u_{\varepsilon}\right|^{2}+\frac{c_{k} \tilde{\varepsilon}}{(s-r)^{2 k+2}} \int_{B_{2 R}}\left(\sum_{i=0}^{k-1}\left|D^{i} D_{j} u_{\varepsilon}\right|^{2}+\left|D^{k} u_{\varepsilon}\right|^{2}\right)
$$

where we remark that the increased power of the factor $(s-r)$ is due to the presence of an additional $D_{j}$-derivative on $\rho^{2 k}$ in $I I I_{2}$. Collecting the above bounds and 
adjusting the constant $c_{k}$ we arrive at

$$
I I I \leq-\frac{\tilde{\varepsilon}}{3} \int_{\Omega^{\prime}} \rho^{2 k}\left|D_{j} D^{k} u_{\varepsilon}\right|^{2}+\frac{c_{k} \tilde{\varepsilon}}{(s-r)^{2 k+2}} \int_{B_{2 R}}\left(\sum_{i=0}^{k-1}\left|D^{i} D_{j} u_{\varepsilon}\right|^{2}+\left|D^{k} u_{\varepsilon}\right|^{2}\right)
$$

Inserting the bounds (4.6), (4.7), (4.8) in (4.5) and using the properties of $\rho$ we get for each $1 \leq j \leq n$ :

$$
\begin{aligned}
& \frac{1}{4} \int_{\Omega^{\prime}} \rho^{2 k}\left\langle D u_{\varepsilon}\right\rangle^{p-2}\left|D_{j} D u_{\varepsilon}\right|^{2}+\frac{\tilde{\varepsilon}}{3} \int_{\Omega^{\prime}} \rho^{2 k}\left|D_{j} D^{k} u_{\varepsilon}\right|^{2} \\
& \leq \frac{c(p, k)}{(s-r)^{2}} \int_{B_{s} \backslash B_{r}}\left\langle D u_{\varepsilon}\right\rangle^{2 q-p}+\frac{c \tilde{\varepsilon}}{(s-r)^{2 k+2}} \int_{B_{2 R}}\left(\sum_{i=0}^{k-1}\left|D_{j} D^{i} u_{\varepsilon}\right|^{2}+\left|D^{k} u_{\varepsilon}\right|^{2}\right) .
\end{aligned}
$$

Adding up these inequalities over $j \in\{1, \ldots, n\}$ and adjusting the constants we arrive at

$$
\begin{aligned}
& \int_{\Omega^{\prime}} \rho^{2 k}\left\langle D u_{\varepsilon}\right\rangle^{p-2}\left|D^{2} u_{\varepsilon}\right|^{2}+\frac{4 \tilde{\varepsilon}}{3} \int_{\Omega^{\prime}} \rho^{2 k}\left|D^{k+1} u_{\varepsilon}\right|^{2} \\
& \leq \frac{c(n, p, k)}{(s-r)^{2}} \int_{B_{s} \backslash B_{r}}\left\langle D u_{\varepsilon}\right\rangle^{2 q-p}+\frac{A(\varepsilon)}{(s-r)^{2 k+2}},
\end{aligned}
$$

where $A(\varepsilon)$ is independent of $r, s$ and where, by a suitable version of the GagliardoNirenberg interpolation inequality,

$$
A(\varepsilon) \rightarrow 0 \quad \text { as } \quad \varepsilon \searrow 0
$$

Omitting the second term, involving $(k+1)$-th order derivatives, on the left-hand side, the above inequality reduces to

$$
\int_{\Omega^{\prime}} \rho^{2 k}\left\langle D u_{\varepsilon}\right\rangle^{p-2}\left|D^{2} u_{\varepsilon}\right|^{2} \leq \frac{c(n, p, k)}{(s-r)^{2}} \int_{B_{s} \backslash B_{r}}\left\langle D u_{\varepsilon}\right\rangle^{2 q-p}+\frac{A(\varepsilon)}{(s-r)^{2 k+2}} .
$$

Now, taking into account estimate (4.1), an elementary calculation implies that

$$
\left|D\left(\rho^{k} V\left(D u_{\varepsilon}\right)\right)\right|^{2} \leq c(p)\left[\rho^{2 k}\left\langle D u_{\varepsilon}\right\rangle^{p-2}\left|D^{2} u_{\varepsilon}\right|^{2}+k^{2} \rho^{2 k-2}|D \rho|^{2}\left|V\left(D u_{\varepsilon}\right)\right|^{2}\right]
$$

Therefore, by virtue of estimate (4.10) and by the Sobolev Embedding Theorem, 
we obtain

$$
\begin{aligned}
& \left(\int_{\Omega^{\prime}}\left|\rho^{k} V\left(D u_{\varepsilon}\right)\right|^{\frac{2 n}{n-2}}\right)^{\frac{n-2}{n}} \leq c \int_{\Omega^{\prime}}\left|D\left(\rho^{k} V\left(D u_{\varepsilon}\right)\right)\right|^{2} \\
& \leq \frac{c(n, N, p, k)}{(s-r)^{2}} \int_{B_{s} \backslash B_{r}}\left\langle D u_{\varepsilon}\right\rangle^{2 q-p}+\frac{c(n, N, p, k)}{(s-r)^{2}} \int_{B_{s} \backslash B_{r}}\left|V\left(D u_{\varepsilon}\right)\right|^{2} \\
& +\frac{A(\varepsilon)}{(s-r)^{2 k+2}} \\
& \leq \frac{c(n, N, p, k)}{(s-r)^{2}} \int_{B_{S} \backslash B_{r} \cap\left\{\left|D u_{\varepsilon}\right| \leq \mu\right\}}\left\langle D u_{\varepsilon}\right\rangle^{2 q-p} \\
& +\frac{c(n, N, p, k)}{(s-r)^{2}} \int_{B_{s} \backslash B_{r} \cap\left\{\left|D u_{\varepsilon}\right|>\mu\right\}}\left\langle D u_{\varepsilon}\right\rangle^{2 q-p} \\
& +\frac{c(n, N, p, k)}{(s-r)^{2}} \int_{B_{s} \backslash B_{r}}\left|V\left(D u_{\varepsilon}\right)\right|^{2}+\frac{A(\varepsilon)}{(s-r)^{2 k+2}} \\
& \leq \frac{c(n, N, p, k, \mu)}{(s-r)^{2}} \int_{B_{s} \backslash B_{r}}\left(1+\left|V\left(D u_{\varepsilon}\right)\right|^{2}\right)^{\frac{2 q-p}{p}} \\
& +\frac{c(n, N, p, k)}{(s-r)^{2}} \int_{B_{s} \backslash B_{r}}\left|V\left(D u_{\varepsilon}\right)\right|^{2}+\frac{A(\varepsilon)}{(s-r)^{2 k+2}}
\end{aligned}
$$

We can write

$$
\frac{p}{2 q-p}=\frac{\theta}{\frac{n}{n-2}}+\frac{1-\theta}{\frac{q}{p}},
$$

where, since $p<q<p \frac{n}{n-1}$,

$$
\theta=\frac{q-p}{2 q-p} \times \frac{p n}{p n-q(n-2)} \in(0,1)
$$

(note that the case $p=q$ does not require these arguments). Hölder's inequality yields

$$
\begin{aligned}
\int_{B_{S} \backslash B_{r}} & \left|V\left(D u_{\varepsilon}\right)\right|^{\frac{2(2 q-p)}{p}} \\
\leq & \left(\int_{B_{S} \backslash B_{r}}\left|V\left(D u_{\varepsilon}\right)\right|^{\frac{2 n}{n-2}}\right)^{\frac{\theta(n-2)}{n} \frac{2 q-p}{p}}\left(\int_{B_{S} \backslash B_{r}}\left|V\left(D u_{\varepsilon}\right)\right|^{\frac{2 q}{p}}\right)^{\frac{(1-\theta)(2 q-p)}{q}}
\end{aligned}
$$


Inserting the previous inequality in (4.11), we obtain

$$
\begin{aligned}
& \int_{\Omega^{\prime}}\left|\rho^{k} V\left(D u_{\varepsilon}\right)\right|^{\frac{2 n}{n-2}} \\
\leq & \frac{c(n, N, p, k, \mu)}{(s-r)^{\frac{2 n}{n-2}}}\left(\int_{B_{s} \backslash B_{r}}\left|V\left(D u_{\varepsilon}\right)\right|^{\frac{2 n}{n-2}}\right)^{\theta \frac{2 q-p}{p}}\left(\int_{B_{s} \backslash B_{r}}\left|V\left(D u_{\varepsilon}\right)\right|^{\frac{2 q}{p}}\right)^{\frac{(1-\theta)(2 q-p)}{q} \frac{n}{n-2}} \\
& +\frac{c(n, N, p, k)}{(s-r)^{\frac{2 n}{n-2}}}\left(\int_{B_{s} \backslash B_{r}}\left|V\left(D u_{\varepsilon}\right)\right|^{2}\right)^{\frac{n}{n-2}}+\frac{c(n, N, p, k, \mu)}{(s-r)^{\frac{2 n}{n-2}}\left|B_{s} \backslash B_{r}\right|^{\frac{n}{n-2}}} \\
& +\frac{\tilde{A}(\varepsilon)}{(s-r)^{\frac{(2 k+2) n}{n-2}}}
\end{aligned}
$$

where we set $\tilde{A}(\varepsilon)=(A(\varepsilon))^{\frac{n}{n-2}}$. Since

$$
\frac{2 q-p}{p} \theta=\frac{(q-p) n}{p n-q(n-2)}<1
$$

for $q<\frac{p n}{n-1}$, it is legitimate to apply Young's inequality with the pair of conjugate exponents

$$
d=\frac{p n-q(n-2)}{(q-p) n} \quad \text { and } \quad d^{\prime}=\frac{1}{2} \frac{p n-q(n-2)}{p n-q(n-1)}
$$

in the second line of (4.12), thus getting

$$
\begin{aligned}
& \int_{B_{r}}\left|V\left(D u_{\varepsilon}\right)\right|^{\frac{2 n}{n-2}} \leq \int_{\Omega^{\prime}}\left|\rho^{k} V\left(D u_{\varepsilon}\right)\right|^{\frac{2 n}{n-2}} \\
& \leq \frac{1}{2}\left(\int_{B_{S} \backslash B_{r}} \mid V\left(D u_{\varepsilon}\right)^{\frac{2 n}{n-2}}\right) \\
& +\frac{c(n, N, p, k, \mu)}{(s-r)^{d^{\prime} \frac{2 n}{n-2}}}\left(\int_{B_{S} \backslash B_{r}}\left|V\left(D u_{\varepsilon}\right)\right|^{\frac{2 q}{p}}\right)^{\frac{(p-q) n+2 q-p}{p n-q(n-1)} \frac{n}{n-2}} \\
& +\frac{c(n, N, p, k)}{(s-r)^{\frac{2 n}{n-2}}}\left(\int_{B_{S} \backslash B_{r}}\left|V\left(D u_{\varepsilon}\right)\right|^{2}\right)^{\frac{n}{n-2}} \\
& +\frac{c(n, N, p, k, \mu)}{(s-r)^{\frac{2 n}{n-2}}}\left|B_{s} \backslash B_{r}\right|^{\frac{n}{n-2}}+\frac{\tilde{A}(\varepsilon)}{(s-r)^{\frac{(2 k+2) n}{n-2}}} .
\end{aligned}
$$


As this estimate is valid for all radii $R \leq r<s \leq 2 R$, we can apply the hole-filling method of Widman. This yields in the usual manner

$$
\begin{aligned}
\int_{B_{R}}\left|V\left(D u_{\varepsilon}\right)\right|^{\frac{2 n}{n-2}} \leq & \frac{c(n, N, p, k, \mu)}{R^{d^{\prime} \frac{2 n}{n-2}}}\left(\int_{B_{2 R}}\left|V\left(D u_{\varepsilon}\right)\right|^{\frac{2 q}{p}}\right)^{\frac{(p-q) n+2 q-p}{p n-q(n-1)} \frac{n}{n-2}} \\
& +\frac{c(n, N, p, k)}{R^{\frac{2 n}{n-2}}}\left(\int_{B_{2 R}}\left|V\left(D u_{\varepsilon}\right)\right|^{2}\right)^{\frac{n}{n-2}} \\
& +c(n, N, p, k, \mu) R^{n}+\frac{\tilde{A}(\varepsilon)}{R^{\frac{(2 k+2) n}{n-2}}} .
\end{aligned}
$$

From estimate (4.14), through the higher integrability of Theorem 1.2, it follows that $V\left(D u_{\varepsilon}\right)$ is bounded in $\mathrm{L}^{\frac{2 n}{n-2}}\left(B_{R}, \mathbb{R}^{N \times n}\right)$ uniformly as $\varepsilon \searrow 0$ and so, by the arbitrariness of the ball $B_{2 R}\left(x_{0}\right) \subset \Omega^{\prime}$ and a simple covering argument, we conclude that $V\left(D u_{\varepsilon}\right)$ is bounded in $\mathrm{L}_{\text {loc }}^{\frac{2 n}{n-2}}\left(\Omega^{\prime}, \mathbb{R}^{N \times n}\right)$. In view of (4.1) and (4.10) it then also follows that $\left(V\left(D u_{\varepsilon}\right)\right)$ is bounded in $\mathrm{W}_{\text {loc }}^{1,2}\left(\Omega^{\prime}, \mathbb{R}^{N \times n}\right)$ uniformly as $\varepsilon \searrow 0$. Therefore, we conclude by passing to the limits as $\varepsilon \searrow 0$, using also compactness of the Sobolev embedding on the right-hand side and Fatou's Lemma on the lefthand side, that

$$
\begin{aligned}
\int_{B_{R}}|V(D u)|^{\frac{2 n}{n-2}} \leq & \frac{c(n, N, p, \mu)}{R^{d^{\prime} \frac{2 n}{n-2}}}\left(\int_{B_{2 R}}|V(D u)|^{\frac{2 q}{p}}\right)^{\frac{(p-q) n+2 q-p}{p n-q(n-1)} \frac{n}{n-2}} \\
& +\frac{c(n, N, p)}{R^{\frac{2 n}{n-2}}}\left(\int_{B_{2 R}}|V(D u)|^{2}\right)^{\frac{n}{n-2}} \\
& +c(n, N, p, \mu) R^{n}
\end{aligned}
$$

and

$$
\begin{aligned}
\int_{B_{R}}|D(V(D u))|^{2} \leq & \frac{c(n, N, p, \mu)}{R^{d^{\prime} \frac{2 n}{n-2}}}\left(\int_{B_{2 R}}|V(D u)|^{\frac{2 q}{p}}\right)^{\frac{(p-q) n+2 q-p}{p n-q(n-1)} \frac{n}{n-2}} \\
& +\frac{c(n, N, p)}{R^{\frac{2 n}{n-2}}}\left(\int_{B_{2 R}}|V(D u)|^{2}\right)^{\frac{n}{n-2}} \\
& +c(n, N, p, \mu) R^{n}
\end{aligned}
$$




\section{References}

[1] E. ACERBI and N. FusCO, Partial regularity under anisotropic $(p, q)$ growth conditions, J. Differential Equations 107 (1994), 46-67.

[2] M. Bildhauer, "Convex Variational Problems. Linear, Nearly Linear and Anisotropic Growth Conditions", Lecture Notes in Mathematics, Vol. 1818. Springer-Verlag, Berlin, 2003.

[3] M. Carozza, J. Kristensen and A. Passarelli di Napoli, Higher differentiability of minimizers of convex variational integrals, Ann. Inst. H. Poincaré Anal. Non Linéaire 28 (2011), 395-411.

[4] M. Carozza, G. Moscariello and A. Passarelli di NAPOli, Regularity results via duality for minimizers of degenerate functionals, Asympt. Anal. 44 (2005), 221-235.

[5] M. CAROZZA and A. PASSARELli DI NAPOLI, Regularity for minimizers of degenerate elliptic functionals, J. Nonlinear Convex Anal. 7 (2006), 375-383.

[6] I. Ekeland and R. Temam. "Convex Analysis and Variational Problems", Classics in Applied Mathematics 28, SIAM, Philadelphia, 1999.

[7] L. Esposito, F. LEONETTI and G. Mingione, Higher integrability for minimizers of integral functionals with $(p, q)$ growth. J. Differential Equations 157 (1999), 414-438.

[8] L. Esposito, F. LEONETTI and G. MingIONE, Regularity results for minimizers of irregular integrals with $(p, q)$ growth. Forum Mathematicum 14 (2002), 245-272.

[9] L. Esposito, F. Leonetti and G. Mingione, Sharp regularity for functionals with $(p, q)$ growth, J. Differential Equations 204 (2004), 5-55.

[10] I. FONSECA and N. FUSCO, Regularity results for anisotropic image segmentation models, Ann. Scuola Norm. Sup. Pisa Cl. Sci. 24 (1997), 463-499.

[11] I. FonsECA, J. MALÝ and G. MingIONE, Scalar minimizers with fractional singular sets, Arch. Ration. Mech. Anal. 172 (2004), 295-307.

[12] N. FUSCO and C. SBORDONE, Higher integrability of the gradient of minimizers of functionals with nonstandard growth conditions, Comm. Pure Appl. Math. 43 (1990), 673-683.

[13] M. GiaquinTA, Growth conditions and regularity, a counterexample, Manuscripta Math. 59 (1987), 245-248.

[14] E. GIUSTI, "Direct Methods in the Calculus of Variations", World Scientific, 2003.

[15] M.C. Hong, Some remarks on the minimizers of variational integrals with nonstandard growth conditions, Boll. Un. Mat. Ital. A (7) 6 (1992), 91-101.

[16] J. KRISTENSEN and C. MELCHER, Regularity in oscillatory nonlinear elliptic systems, Math. Z. 260 (2008), 813-847.

[17] J. KRISTENSEN and G. MingIONE, The singular set of minima of integral functionals, Arch. Ration. Mech. Anal. 180 (2006), 331-398.

[18] P. MARCELlini, Un example de solution discontinue d'un probléme variationnel dans le cas scalaire, Preprint Ist. U. Dini, Firenze, 1987-88.

[19] P. MarCELlini, Regularity of minimizers of integrals of the calculus of variations with non-standard growth conditions, Arch. Ration. Mech. Anal. 105 (1989), 267-284.

[20] P. MARCELlini, Regularity and existence of solutions of elliptic equations with $(p, q)$ growth conditions, J. Differential Equations 90 (1991), 1-30.

[21] P. MARCELLINI, Everywhere regularity for a class of elliptic systems without growth conditions, Ann. Scuola Norm. Sup. Pisa, Cl. Sci. 23 (1996), 1-25.

[22] P. MARCELlini and G. PAPI, Nonlinear elliptic systems with general growth, J. Differential Equations 221 (2006), 412-443.

[23] G. MingIone, Regularity of minima: an invitation to the dark side of the calculus of variations, Appl. Math. 51 (2006), 355-426.

[24] A. Passarelli Di NAPOLI and F. SiePe, A regularity result for a class of anisotropic systems, Rend. Ist. Mat. Univ. Trieste 28 (1996), 13-31.

[25] T. SCHMIDT, Regularity of minimizers of $W^{1, p}$-quasiconvex variational integrals with ( $p, q)$-growth, Calc. Var. Partial Differential Equation 32 (2008), 1-24. 
[26] T. SCHMIDT Regularity of relaxed minimizers of quasiconvex integrals variational integrals with $(p, q)$-growth, Arch. Ration. Mech. Anal. 193 (2009), 311-337.

[27] V. Š VERÁK and X. YAN, Non-Lipschitz minimizers of smooth uniformly convex variational integrals, Proc. Nat. Acad. Sci. USA 99 (2002), 15269-15276.

[28] L. TARTAR, "An Introduction to Sobolev Spaces and Interpolation Spaces", Lecture Notes of the Unione Math. Italiana 3, Springer-Verlag Berlin Heidelberg, 2007.

[29] W.P. ZiEMER, 'Weakly Differentiable Functions", Graduate Texts in Maths., Vol. 120, Springer-Verlag, 1989.

\author{
Università del Sannio \\ Dipartimento di Ingegneria \\ Corso Garibaldi \\ 82100 Benevento, Italia \\ carozza@unisannio.it \\ Mathematical Institute \\ University of Oxford \\ Andrew Wiles Building \\ Radcliffe Observatory Quarter \\ Woodstock Road \\ Oxford OX2 6GG, England \\ kristens@maths.ox.ac.uk \\ Università di Napoli 'Federico II' \\ Dipartimento di Matematica \\ e Applicazioni 'R. Caccioppoli' \\ Via Cintia \\ 80126 Napoli, Italia \\ antpassa@unina.it
}

